\title{
Surface processing of optical glasses with 34 fs pulses: ablation thresholds and crater shape
}

\author{
Andrásik Attila ${ }^{1,2}$, Flender Roland ${ }^{1}$, Budai Judit ${ }^{1}$, Szörényi Tamás ${ }^{1}$, Hopp Béla ${ }^{1}$ \\ ${ }^{1}$ Department of Optics and Quantum Electronics, University of Szeged, H-6720 Szeged, Dóm tér 9., \\ Hungary \\ ${ }^{2}$ Department of Photonics and Laser Research, Interdisciplinary Excellence Centre, University of \\ Szeged, Szeged, Hungary
}

DOI: https://doi.org/10.14232/kvantumelektronika.9.2

\section{Introduction}

Since the advent of availability of high-energy fs pulses [1], the peculiarities of the interaction of ultrashort laser pulses with matter are intensively studied. One effect attracting interest is that due to the ionization of transparent dielectrics, dense plasma is created on the surface with enhanced reflectivity [2-5] weakening the energy coupling efficiency, diminishing thereby the effectivity of the material removal [6,7]. For that reason, comprehensive description of ionization induced effects is highly required.

Due to its extraordinary optical and material quality, fused silica is the favourite material of the fs ablation studies [6, 8-10,11-20]. Commercial multicomponent borosilicate and crown glasses received astonishingly little attention, even though they may serve as cheaper and more accessible alternatives.

Borofloat, BK7 and B270 optical glasses were studied in this work reporting an analysis of the evolution of the morphology and main geometrical properties of the ablated craters generated by ultrashort, high-intensity pulses. The evolution of the crater shape and depth is investigated in the context of accompanying ionization of the targets and a model based on multiphoton absorption of the incoming processing beam.

\section{Experimental}

A scheme about the locally developed arrangement to conduct the experiments is seen in Fig. 1. The driving laser for the presented PM experiment was a locally-developed Ti:Sapphire based chirped pulse amplification (CPA) system (TeWaTi laser system, [21]), which are able to deliver laser pulses with $1 \mathrm{~mJ}$ pulse energy, $34 \mathrm{fs}$ pulse duration at $800 \mathrm{~nm}$ central wavelength. Uncoated optical glass pieces placed at an angle of incidence of $45^{\circ}$ were applied as targets. Shot-to-shot repositioning of the target using translation stages served to ensure the ablation of pristine surface areas. The beam was focused onto the target with a parabolic mirror. The reflected beam was re-focused by a lens to totally overlap with the sensor area of the photodiode PD. The actual diameter of the beam on the sample surface was determined according to the formula:

$$
D^{2}=2 w^{2} \ln \left(F / F_{t h}\right),
$$


where $w$ is the $1 / \mathrm{e}^{2}$ beam radius, while $F$ and $F_{\text {th }}$ stand for the peak and ablation threshold fluences, respectively [22]. Both the diameter-regression technique (DR) based on the application of Eq. (1), and the multiphoton absorption-based fitting of the depth vs. fluence functions (MA), [9, 23], applying

$$
d_{\max }=\frac{\tau^{m-1}}{\left[(m-1) \alpha_{m}(1-R)^{m-1}\right]} *\left(\frac{1}{F_{t h, d e p}^{m-1}}-\frac{1}{F^{m-1}}\right),
$$

with $d_{\max }$ as depth, $m$ as the order, $\alpha_{m}$ as the non-linear absorption coefficient, $\tau$ as the pulse duration (actually $34 \mathrm{fs}$ ) and $R$ as the measured linear reflection coefficient were used to derive the ablation thresholds.

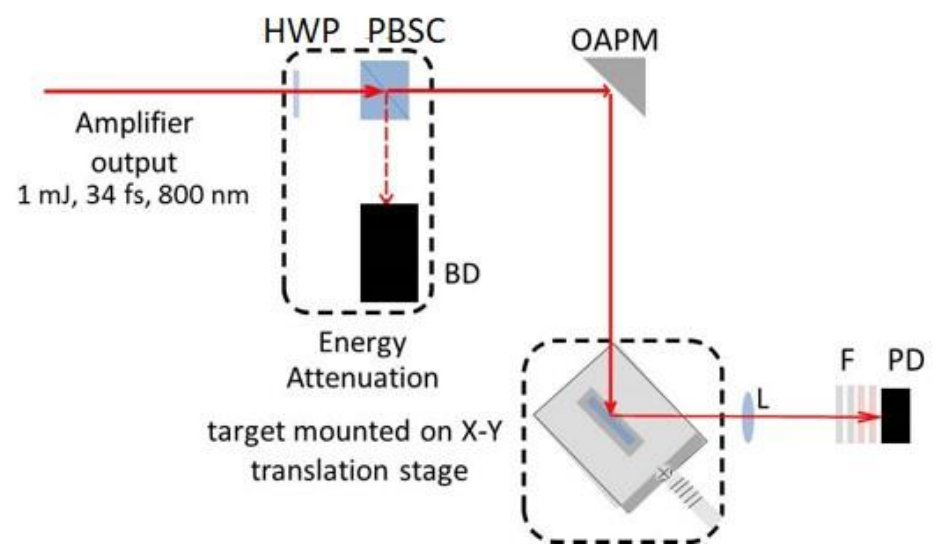

Fig. 1 Scheme of the setup (see details also in [24, 25]). HWP: half-wave plate; PBSC: polarization beam-splitter cube; BD: beam dumper; OAPM: off-axis parabolic mirror; L: focusing bi-convex lens; F: filters; PD: photodiode

\section{Results}

The profilometer traces recorded along the minor axes shown in Fig. 2 reveal that the shape of the craters follows reliably the Gaussian-like energy distribution of the processing beam for all three glasses with small differences in the morphology. For Borofloat and BK7 both the shape and the roughness of the craters remain essentially unchanged with increasing fluence. With the contour remaining smooth but the shape changing, B270 exhibits different evolution as a function of fluence: a bump evolves as the fluence increases resulting in slightly smaller depth at the center as compared to the maximum depth measured towards the edges.

In Fig. 3 the evolution of the diameter and the depth of the ablated craters are plotted as a function of fluence together with the part of the processing beam reflected from the irradiated area measured as the photodiode signal for the three glasses. In the whole fluence range investigated the welldocumented logarithmic dependence (formulated in Eq. (1)) characterizes the evolution of the diameter values above threshold, reaching values around $45 \mu \mathrm{m}$ at $30 \mathrm{~J} / \mathrm{cm}^{2}$ for all three glasses. Contrary to the diameters the depths increase with increasing fluence and show saturation starting at slightly different fluences: above 20, 17 and $18 \mathrm{~J} / \mathrm{cm}^{2}$ for Borofloat, BK7 and B270, respectively.

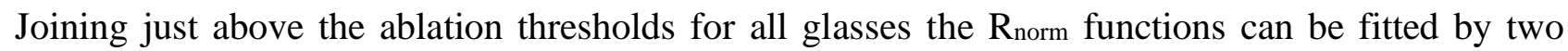
straight sections with different slopes ( $a_{p}$ and $a_{t}$ ) (Fig. 3 g) - i)) marking a change in the optical response of the irradiated area at the breakpoint fluence. In the steep increase in the $\mathrm{R}_{\text {norm }}$ above the breakpoint the emergence of a plasma mirror materializes [25]. 
With values of $9.5,10$ and $8.0 \mathrm{~J} / \mathrm{cm}^{2}$ for Borofloat, BK7 and B270, respectively, the breakpoints differ only slightly for the three glasses and correlate well with the ablation thresholds [26], which supports

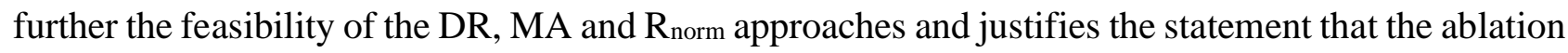
characteristics of the glasses investigated are rather similar.

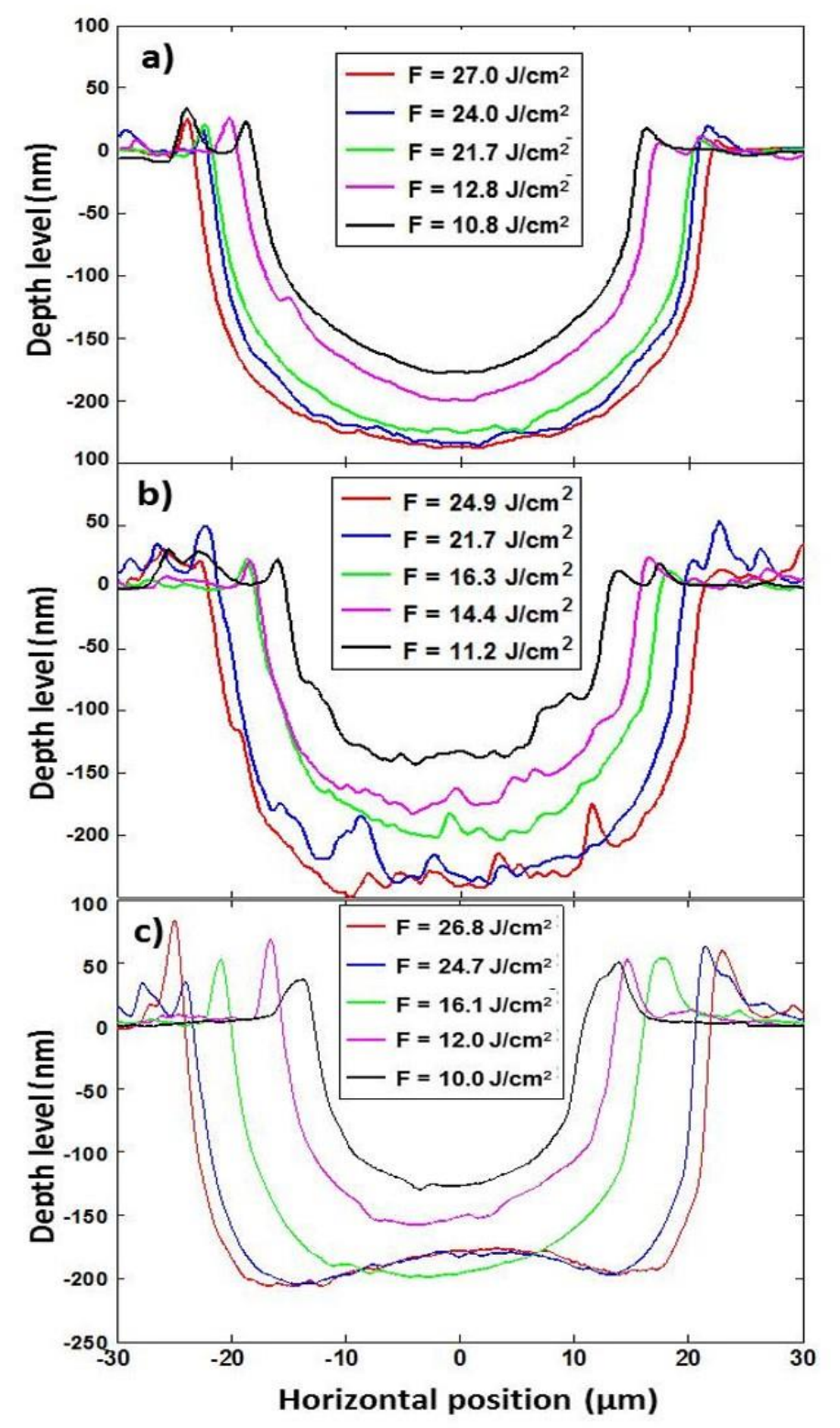

Fig. 2 Evolution of the cross sections along the minor axes of the craters ablated into a) Borofloat $b$ ) BK7 and c) B270 glasses. Beam radii on the surface: 25.1, 26.1 and $24.83 \mu \mathrm{m}$, respectively. 


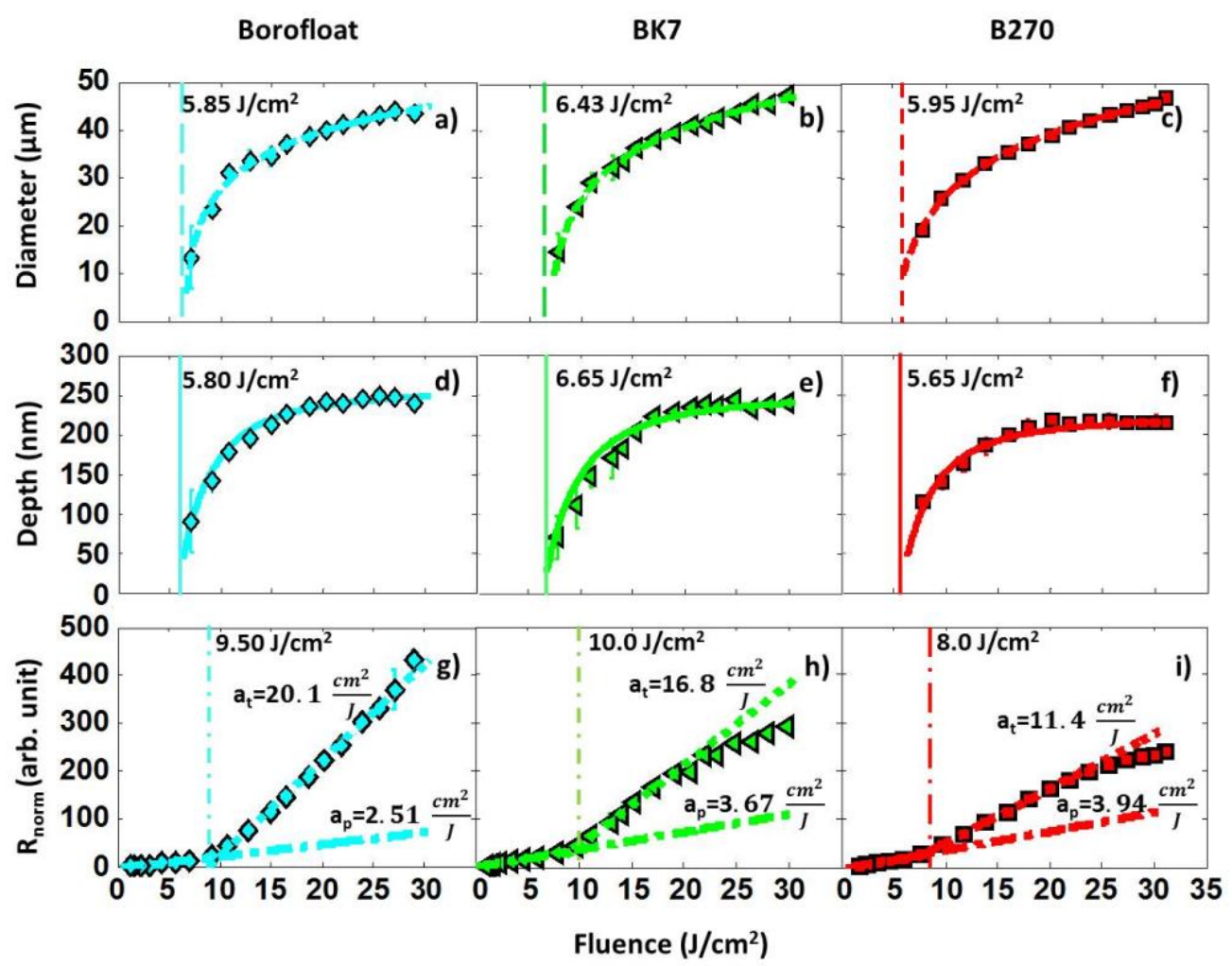

Fig. 3 Ablation and reflection characteristics of the glasses. Dashed curves in a)-c) are logarithmic fits, while continuos curves denote in d)-f) the MA fits according to Eq. (2). Dashed and continuous vertical lines mark the ablation thresholds derived from DR and MA fits, respectively. $R_{\text {norm }}$ stands for normalized reflectivity. Dash-dotted vertical lines indicate the breakpoints between the two straight sections of the reflectivity, marking the onset of plasma formation. Dash-dotted and dotted lines with slopes $a_{p}$ and $a_{t}$ show the difference between the evolution of permanent and transient reflectivities in $\mathrm{g}$ )-i).

\section{Discussion}

Above a certain fluence only minor differences were observed in the shape of the craters ablated into Borofloat, BK7 and B270 glasses: a bump emerged in the middle of the bottom of the crater when processing B270 glass with pulses of fluences exceeding $24 \mathrm{~J} / \mathrm{cm}^{2}$ (cf. Fig. $2 \mathrm{c}$ ) which is an intriguing phenomenon not mentioned for fs ablation of glasses. Uteza [6] describes such bump formation at the bottom of the craters ablated into a fused silica target with $7 \mathrm{fs}$ and $30 \mathrm{fs}$ pulses developing with increasing fluence above $10 \mathrm{~J} / \mathrm{cm}^{2}$. As an explanation for the bump formation transient reflectivity enhancement in the central part of the irradiated area [6] and saturation of nonlinear absorption at high fluence [7] could be considered. Concerning the crater dimensions, the logarithmic diameter vs. fluence functions and the saturation in depths compare favorably with the results recorded for glasses $[23,26,27]$.

Three-photon absorption was considered when fitting Eq. (2) to the measured depth values (Fig. $3 \mathrm{~d}$ )f)) [23]. The fit resulted in three photon absorption coefficients, $\alpha_{3}: 6.45,6.35$ and $8.28 \times 10^{-25} \mathrm{~cm}^{3} / \mathrm{W}^{2}$ for Borofloat, BK7 and B270, respectively. Comparing these figures with data available in the relevant literature we found that the $\alpha_{3}$ values derived by Grehn [23] were two orders of magnitude higher than ours, which has several possible explanations: i) the absorption-shielding effect of the plasma might be reflected in the lower $\alpha_{3}$ values obtained by us, ii) pulse shortening from ablating 
with much shorter pulses as compared to Grehn's could result in less effective ablation [18, 27], and iii) our results refer to an intensity domain with increasing contribution from tunneling while in the case described by Grehn the three-photon absorption is more dominant.

\section{Conclusions}

The ablation threshold values derived from both the diameter- and multiphoton absorption-based fits proved to be equal within experimental error lying in the $6.1 \pm 0.55 \mathrm{~J} / \mathrm{cm}^{2}$ domain with overlapping confidence intervals. The ablated crater shapes of Borofloat and BK7 behave similarly, while a bump emerges in the middle of the bottom of the crater dug into the B270 glass. The onset of plasma formation marked by the optical fluence thresholds well correlating with the ablation thresholds, is defined by the change in the reflectivity from the processed surface. The three photon absorption coefficients derived from the multiphoton absorption-based fit are also similar lying in the same magnitude for the glasses investigated. Along with the findings related to the ablation parameters, nonlinear absorption coefficients and reflectivity evolution, it can be stated that the fluence dependence of the ablation characteristics is the same with minor differences in the optical response of the glasses.

\section{Acknowledgements}

The project has been supported by the European Union, co-financed by the European Social Fund. EFOP-3.6.2-16-2017-00005, "Ultrafast physical processes in atoms, molecules, nanostructures and biological systems “.

This work has also been supperted by ELI-ALPS, ELI-HU Non-Profit Ltd. H-6720 Szeged, Dugonics tér 13; GINOP-2.3.6-15-2015-00001 and ELI_GINOP_4_0125 and The Ministry of Human Capacities, Hungary; grant 20391-3/2018/FEKUSTRAT.

The authors wish to thank Ádám Börzsönyi, Mikhail Kalashnikov and Csaba Vass from ELI-ALPS, ELI-HU Non-Profit Ltd. H-6720 Szeged, Dugonics tér 13 for valuable discussions.

\section{References}

[1] D. Strickland, G. Mourou, Opt. Comm. 56, 3 (1985)

https://doi.org/10.1016/0030-4018(85)90120-8

[2] H.C. Kapteyn, M.M. Murname, A. Szoke, R. W. Falcone, Opt. Lett. 16, 7 (1991)

https://doi.org/10.1364/OL.16.000490

[3] Ch. Ziener, P.S. Foster, E.J. Divall, C.J. Hooker, M.H.R. Hutchinson, A.J. Langley, D. Neely, J. of Appl. Phys. 93, 768 (2003)

https://doi.org/10.1063/1.1525062

[4] G. Doumy, F. Quéré, O. Gobert, M. Pedrix, Ph. Martin, P. Audebert, J.C. Gauthier, J.-P. Geindre, T. Wittmann, Phys. Rev. E 69, 026402 (2004)

https://doi.org/10.1103/PhysRevE.69.026402

[5] B. Dromey, S. Kar, M. Zepf, P. Foster, Rev. Sci. Instr. 75, 645 (2004)

https://doi.org/10.1063/1.1646737 
[6] O. Utéza, N. Sanner, B. Chimier, A. Brocas, N. Varkentina, M. Sentis, P. Lassonde, F. Légaré, J. C. Kieffer, Appl. Phys. A 105, 131-141 (2011) https://doi.org/10.1007/s00339-011-6469-y

[7] M. Lebugle, N. Sanner, O. Utéza, M. Sentis, Appl. Phys. A 114, (2014) https://doi.org/10.1007/s00339-013-8153-X

[8] J. Krüger, W. Kautek, M. Lenzner, S. Sartania, C. Spielmann, F. Krausz, Proc. of SPIE Las. Applications in Microel. and Optoel. Manufacturing II (1997) https://doi.org/10.1117/12.273740

[9] D. Puerto, J. Siegel, W. Gawelda, M. Galvan-Sosa, I. Ehrentraut, J. Bonse J. Solis, J. Opt. Soc. Am. B 27, 5 (2010) https://doi.org/10.1364/JOSAB.27.001065

[10] L. Hoffart, P. Lassonde, F. Légaré, F. Vidal, N. Sanner, O. Utéza, M. Sentis, J.-C. Kieffer, I. Brunette, Opt. Exp. 19, 1 (2011) https://doi.org/10.1364/OE.19.000230

[11] A. Rosenfeld, D. Ashkenasi, H. Varel, M. Wahmer, E. E. B. Campbell, Appl. Surf. Sci. 127-129 (1998)

https://doi.org/10.1016/S0169-4332(97)00613-2

[12] M. Lenzner, J. Krüger, W. Kautek, F. Krausz, Appl. Phys. A 68, 3 (1999) https://doi.org/10.1007/s003390050906

[13] A. P. Joglekar, H. Liu, G. J. Spooner, E. Meyhöfer, G. Mourou A. J. Hunt, Appl. Phys. B 77, 1 (2003) https://doi.org/10.1007/s00340-003-1246-Z

[14] D. Giguére, G. Olivié, F. Vidal, S. Toetsch, G. Girard, T. Ozaki, J. C. Kieffer, J. Opt. Soc. Am. A 24, 6 (2007) https://doi.org/10.1364/JOSAA.24.001562

[15] D. F. Farson, H. W. Choi, B. Zimmerman, J. K. Steach, J. J. Chalmers, S. V. Olesik, L. J. Lee, J. Micromech. Microeng. 18, 3 (2008) https://doi.org/10.1088/0960-1317/18/3/035020

[16] N. Sanner, B. Bussiere, O. Utéza, A. Leray, T. Itina, M. Sentis, J.Y. Natoli, M. Commandré, Proc. of SPIE. 6881 (2008) https://doi.org/10.1117/12.762767

[17] N. Sanner, O. Utéza, B. Bussiere, G. Coustillier, A. Leray, T. Itina, M. Sentis, Appl. Phys. A 94, 4 (2009) https://doi.org/10.1007/s00339-009-5077-6

[18] B. Chimier, O. Utéza, N. Sanner, M. Sentis, T. Itina, P. Lassonde, F. Légaré, F. Vidal, J.C. Kieffer, Phys. Rev. B 84, 9 (2011) https://doi.org/10.1103/PhysRevB.84.094104

[19] M. H. Shaheen, J. E. Gagnon, B. J. Fryer, Laser Phys. 24, 10 (2014) https://doi.org/10.1088/1054-660X/24/10/106102 
[20] S.-Z. Xu, C.-Z. Yao, W. Liao, X.-D. Yuan, T. Wang, X.-T. Zu, Nucl. Instr. and Methods in Phys. Res. B 385 (2016)

https://doi.org/10.1016/j.nimb.2016.06.016

[21] Webpage of University of Szeged, Department of Optics and Quantumelectronics: http://opt.physx.u-szeged.hu/node/45 (accessed at 11.26. 2020)

[22] J. M. Liu, Opt. Lett. 7, 5 (1982)

https://doi.org/10.1364/OL.7.000196

[23] M. Grehn, T. Seuthe, M. Höfner, N. Griga, C. Theiss, A. Mermillod-Blondin, M. Eberstein, H. Eichler and J. Bonse, Opt. Mat. Exp. 4, 4 (2014)

https://doi.org/10.1364/OME.4.000689

[24] A. Andrásik, R. Flender, J. Budai, T. Szörényi, B. Hopp, Appl. Phys. A. 126, 936 (2020)

https://doi.org/10.1007/s00339-020-04122-z

[25] A. Andrásik, R. Flender, J. Budai, T. Szörényi, B. Hopp, Opt. Mater. Exp. 10, 2 (2020)

https://doi.org/10.1364/OME.380294

[26] I. Mirza, N. M. Bulgakova, J. Tomástík, V. Michálek, O. Haderka, L. Fekete, T. Mocek, Sci. Rep. 6 (2016)

https://doi.org/10.1038/srep39133

[27] A. Ben-Yakar, R. L. Byer, J. of Appl. Phys. 96, 9 (2004)

https://doi.org/10.1063/1.1787145

[28] T. Itina, O. Utéza, N. Sanner, M. Sentis, J. of Optoel. and Adv. Mat. 12, 3 (2010) 